\title{
A temporal-focused trustworthiness to enhance trust-based recommender systems
}

\begin{abstract}
Collaborative Filtering (CF) is the most successful technology for recommender systems. The technology does not rely on actual content of the items, but instead requires users to indicate preferences, most commonly in the form of ratings. While CF is known for its traditional problems such as cold-start, sparsity and modest accuracy, a trust-based CF has been previously proposed to solve such issues by focusing on trust values among the users. Nonetheless, all existing trust-based approaches use trust as a factor independent from scope, whether explicit or implicit. We argue that trustworthiness should not be the same across all conditions; hence the trust values should change to suit certain scope or focused area. To validate the proposed temporal-focused trustworthiness in this paper, we propose a novel pheromone-based approach to calculate trustworthiness by focusing on time factor. Implementation of the proposed approach is hoped to reduce cold-start and sparsity as well as improve accuracy of the recommendation results.
\end{abstract}

Keyword: Recommender systems; Collaborative filtering; Trust; Temporal-focused; Social tagging systems 